\title{
An Augmented Virtuality Based Solar Energy Power Calculator in Electrical Engineering
}

\author{
http://dx.doi.org/10.3991/ijep.v5i1.3841 \\ C. Onime ${ }^{1}$, J. Uhomoibhi $^{2}$ and E. Pietrosemoli ${ }^{1}$ \\ ${ }^{1}$ Abdus Salam International Centre for Theoretical Physics (ICTP), Trieste, Italy \\ ${ }^{2}$ University of Ulster, Belfast, Northern Ireland
}

\begin{abstract}
It is becoming increasingly important to include information about power generation from renewable energy sources in the training of electrical engineers. Solar energy is arguably the most common renewable energy source in use today. Providing practical hands-on training on solar energy power generation today requires the use of photovoltaic panel devices which are used for transforming solar energy into electrical energy.
\end{abstract}

In many developing countries, practical hands-on training on solar power generation is limited due to the cost of photovoltaic panel devices and so the training consists of theoretical and tutorial classes sometimes supported by remote and virtual laboratories.

This paper presents an augmented virtuality tool where real-time information from a mobile device's sensors is used directly within a virtual or computer generated environment. The tool provides a practical context for hands-on tutorial exercises on solar energy power generation.

Index Terms-Augmented virtuality, computer aid, solar energy, tutorial exercise.

\section{INTRODUCTION}

A good understanding of alternative sources of energy including solar energy is becoming important for the modern electrical engineer. There is a global drive to use new energy sources even at the level of individual home units and it is estimated that at least $20 \%$ of solar panels deployed worldwide are for residential use [1].

In many nations, providing electricity power supply to all citizens even in rural locations is of paramount importance and energy sources such as solar energy power generation are viewed as attractive alternative solutions capable of extending the reach of national power grids especially to island and/or other rural locations [2]. As a result, electrical engineers and professionals in many countries are increasingly, tasked with proving solutions/projects that involve a mix of energy from different sources such as solar, wind and micro-hydro.

\section{A. Educational resources}

Educational resources on solar energy power generation are still quite few and are generally designed for informal and/or professional learning [3]. In many countries, the formal training of electrical engineers is carried out in institutions of higher education and sometimes includes a multi-disciplinary curriculum that may contain courses on science subjects (physics, mathematics, chemistry), along with others such as computer programming [4].

The formal training of engineers typically includes core experimental laboratory work and sometimes other active learning activities such as seminars and capstone projects [5]. Seminars and capstone projects are useful for facilitating interactions with industry especially when such interactions are for solving practical challenges and developing practical know-how (or learning material) [6]. This is demonstrated in the learning material (course) on photovoltaic (solar energy) developed by a consortium of universities and enterprises (academia-industry collaboration) under the E-Learning and Practical Training of Mechatronics and Alternative Technologies in Industrial Community (E-PRAGMATIC), as a lifelong learning resource for the training of European professionals in the electro-mechanical Industry [7].

Apart from the above mentioned course, additional educational/pedagogically resources on photovoltaic/solar energy including simulations, theoretical material and animations are also available from the pveducation.org on-line user portal [8]. Notably, there are already some remote and virtual laboratory experiments on photovoltaic that are relevant for training on solar energy power generation; these tend to focus on measuring performance of devices along with the effects of the immediate physical environment (such as insolation) on the photovoltaic cells [3]. The solar insolation is the total amount of solar energy received at a particular location during a specified time period such as one day; it is often in units of $\mathrm{kWh} / \mathrm{m}^{2}$ per day [8]. The Virtual Physical Laboratory (VPL), part of the virtual and remote laboratory package of the University of Ulster, includes simulations and experiments for fixed and auto-tracked performance (comparative) measurements on solar energy generation systems built using either poly-crystalline or mono-crystalline panels.

For many learners from developing countries, training on solar energy power generation is mostly about the theoretical exercise as the immediate physical environmental conditions in a remote laboratory maybe considerably different from the local physical environment. For solar energy solutions, the physical environment (geographical coordinates and orientation of the solar panel towards the sun) are important as well as the characteristics of the physical photovoltaic device such as operational efficiency and physical dimensions. The expected power output for any solar panel type device may be calculated as a mainly theoretical exercise where the learner is only expected to substitute values into a suitable modeling equation [9]. 


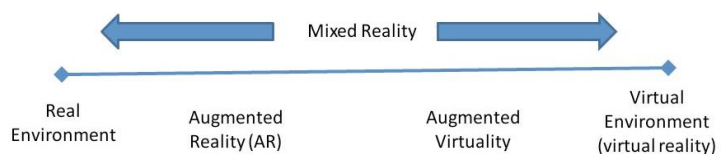

Figure 1. Simple representation of a Reality-Virtuality Continuum

\section{B. Emerging technologies}

An emerging trend in learning and also in on-line experimentation is the use of virtual and augmented reality computing technologies. In virtual reality environments, the goal is to completely replace all physical objects with computer generated equivalents. Sometimes the virtual reality user is required to use/wear special equipment such as hand-wands/head-gear/bodysuits and/or remain in a specially designed environment or room in order to be completely immersed in the virtual environment. Alternatively, mixed reality environments are composed of both computer generated and physical objects. Figure 1 shows the relationship between reality, virtuality as two extremes and augmented reality, augmented virtuality as two forms of mixed reality inbetween the two extremes. Augmented reality is a well known example of the mixed reality environment and in augmented reality, the goal is to blend computer generated information into a physical environment, while in augmented virtuality, the goal is to "blend" the information taken from the physical environment around the viewer into a computer generated environment (virtual world). In other words, the fundamental different between augmented reality and augmented virtuality is that in augmented reality, virtual objects are added onto a view of the real world environment, while in augmented virtuality, real objects (or information) are added into an otherwise virtual environment. As this implies, augmented virtuality has a virtual or computer generated environment, which is used as a reference for all objects. The virtual environment is augmented through the addition of one or more real objects.

A joint research activity between the University of Ulster, Northern Ireland and the International Centre for Theoretical Physics (ICTP), Italy on the use of computer aids in engineering education; named the Computer Aided Engineering Education (CAEE) scheme is concerned with the use of interactive video along with virtual and mixed reality computing technologies for the enhanced, interactive delivery of educational materials in the field of engineering and science while also maintaining existing pedagogical contents and standards. In the CAEE scheme, mixed reality applications running on mobile devices/platforms are used for delivery of hands-on laboratory practical work and tutorial exercises.

This later sections of this paper presents a CAEE augmented virtuality implementation of a solar energy power output calculator that may be used as a practical companion to existing educational resources on solar energy power generation.

\section{IMPLEMENTATION}

The augmented virtuality solar energy power calculator is implemented as an example of the Computer Aided Engineering Education (CAEE) scheme. It is mixed reality tool running on a mobile device (tablets/phones) and is used for the enhanced delivery of practical handson experience on solar energy power generation. Mobile devices especially smart-phones and tablets devices with sensors such as orientation sensors and touch-screens are a cost effective, technologically accepted solution that may be used as acceptable mixed reality viewers. In the resulting augmented virtuality application, real-time information taken directly from the device sensors is blended into a computer generated environment.

\section{A. Theoretial background}

Photovoltaic cells convert light power into electrical power, the resulting output is highly dependent on the incident solar power reaching the surface of a solar panel. Manufacturers of solar panels provide values for several parameters (such as open circuit voltage and short circuit current) useful for determining the operational characteristics of the panel under standard irradiance and temperature conditions at sea level [9]. Since the power is generated only during the hours in which the sun is actually shining, the amount of energy per square meter of solar power captured during one day is the integral of the instantaneous power over 24 hours and is normally expressed in $\mathrm{Wh} / \mathrm{m}^{2}$. This number is often called Peak Sun Hours (PSH), since it is the equivalent of a constant insolation of $1000 \mathrm{~W} / \mathrm{m}^{2}$ shining during the number of hours that would produce the same amount of energy.

In a simplified model of a solar energy based power generation system, four key factors define the resulting electrical energy output, namely: the insolation at the given geographical position, the angular orientation of the solar panel towards the sun, the operational efficiency of the particular solar panel device and the physical dimensions or surface area of the collecting panel. They are related by the equation:

$$
E=G^{*} e^{*} \cos \left|\left(\alpha_{o p}-\alpha\right)\right|
$$

In (1), $E$ is the energy output of solar panel in $\mathrm{Wh} / \mathrm{m}^{2}$, $G$ is the solar insolation value in the given place in $\mathrm{Wh} / \mathrm{m}^{2}$ and $e$ is the panel efficiency, $\alpha_{o p}$ is the optimal angle of inclination (on a first approximation the latitude of the place) and $\alpha$ is the current angle of inclination. If $G$ is expressed in watt-hour per square meter $\left(\mathrm{kWh} / \mathrm{m}^{2}\right)$, the total energy in $\mathrm{kWh}$ captured during one day is obtained by multiplying this number with area of the collecting panel in square meters. Also, in (1), it is necessary to use the absolute value of the angle since the formula is valid for both positive and negative latitudes, but the energy is always positive and related to the absolute value of the difference between the actual and the optimal inclination angle.

\section{B. Main view}

The main view of the augmented virtuality application as shown in Figure 2 is a publicly available solar irradiance map of the whole world. The augmented virtuality definition is derived from the fact that the map has no relationship to the learner's immediate surroundings, and serves to both abstract and confine all objects within it. The solar irradiance map is color coded and the color of a particular location may be translated into the approximate global horizontal insolation value in watt-hour per square meter $\left(\mathrm{Wh} / \mathrm{m}^{2}\right)$ [10] according to the color gradient table shown in the lower left hand corner of Figure 2. Generally, warmer color results in higher values, that is, blue areas have lower values compared to yellow, while red areas have higher values. 


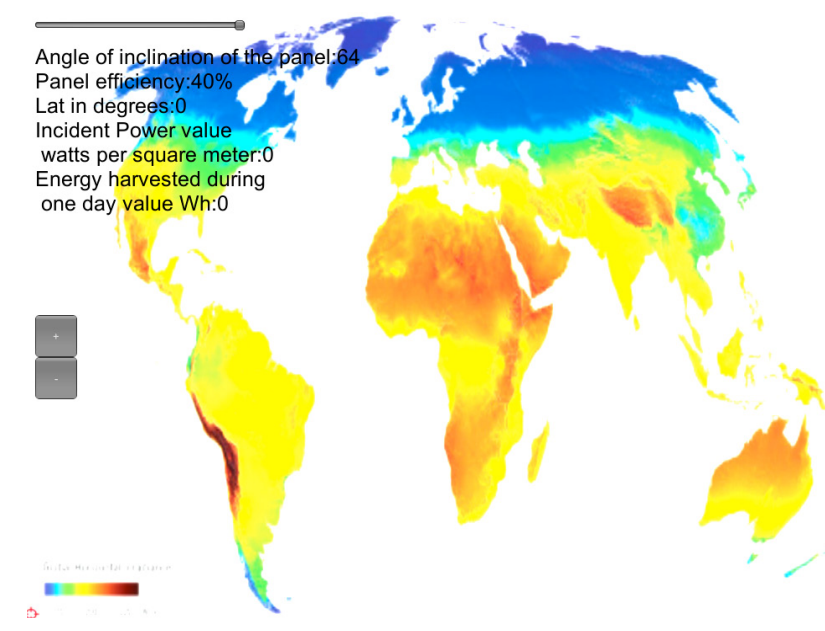

Figure 2. Main view of augmented virtuality application

\section{Interactive inputs and control}

The CAEE implementation is an active learning tool and the learner is required to provide data to the application in an interactive manner. During active learning, the learner is engaged in specially designed activities aimed at reinforcing or delivering some intended learning outcome.

The primary input of geographic location requires the learner to indicate by touching a desired location on the map. This requires the learner to be geographically aware of the location of the intended site of the solar panel. Users quickly learn to appreciate the effect of geographic location on the calculated power output. The application then obtains the geographical latitude information from the indicated point. The current location is indicated by a cross-hair icon that may be moved from one location to another by simply touching the new location on the device display.

A zoom control (shown in the left-hand side of Figure 2 and magnified in the right hand side of Figure 3 ), is implemented as two grey icons/buttons. The button with the + (plus) sign is for magnification (zoom-in) and the lower button with the - (minus) sign is for reduction (zoom-out) of the map. These two buttons play a role in improving the precision of the indicated (selected) location. The zoom control allows users to appreciate the effect of precision on the calculated power output.

A third control object (the slider control) shown in right hand side of Figure 3 allows the user to input the desired panel efficiency. The slider control limits the input range to values between 5 and 40 . For a physical solar panel device, the panel efficiency is typically obtained from the manufacturer specification. Using this control allows users to quickly learn or better appreciate the effect of understanding and interpreting the manufacturer's specifications on the calculated power output.
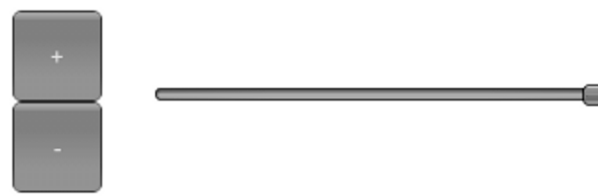

Figure 3. Input controls and panel efficiency control

\author{
Angle of inclination of the panel:25 \\ Panel efficiency: $40 \%$ \\ Lat in degrees:9 \\ Incident Power value \\ watts per square meter:190 \\ Energy harvested during \\ one day value Wh:1752
}

Figure 4. Output from augmented virtuality solar energy calculator

The fourth interactive input from the learner is the current inclination or angular orientation data of a solar panel. For optimal production of energy, solar panels have to be oriented towards the energy source (sunlight) to obtain the maximum power. This highlights the difference between solar panels that are installed at high latitudes (in either hemisphere), which will require steeper inclination angles compared with those installed near the Equator. For an optimal power output, the solar panel should be installed in a location where it will receive maximal incidence of sunlight avoiding objects that could cause shade in its surface at any time of the day. In the application, the angular inclination is read in real-time directly from the mobile device sensors as the learner changes the inclination of the device simulating that of the solar panel. This implies that the mobile device used must include the appropriate hardware sensors. Users quickly learn to appreciate the effect of angular inclination on the calculated power output.

\section{Immediate feedback/output}

As a computer based learning aid, the augmented virtuality solar energy power calculator is able to provide the augmented output or results as immediate feedback to the learner. The augmented output/immediate feedback is based on the various interactive input supplied by the learner.

The output from the software application is composed of 5 values as shown in Figure 4. The first output value is the actual real-time angle of inclination of the mobile device as obtained from the hardware sensors. The second output value is the current panel efficiency which is set by the slider control shown in Figures 3 and 4 . The third value presents the current latitude as determined from the indicated/touched location on the displayed solar map (device display). The fourth value is the global horizontal irradiance at the indicated location computed from the color gradient table and the fifth value shows the computed energy as the energy harvested during one day.

An example of the augmented virtuality solar panel calculator in use is shown in Figure 5 for a location in eastern Nigeria (in the African continent) as indicated by the cross-hairs. The location is situated at about 9 degrees above the Equator, the mean global daily solar irradiance at this place is determined from the map to be $190 \mathrm{~W} / \mathrm{m}^{2}$.

To obtain the total energy captured during one day we must multiply by 24 , the number of hours in one day.

Substituting the values in (1) gives $190 \mathrm{~W} / \mathrm{m}^{2} * 0.4 *$ $\cos |(9-25)|=72.78 \mathrm{~W} / \mathrm{m}^{2} \approx 73 \mathrm{~W} / \mathrm{m}^{2} * 24 \mathrm{~h}=1752$ $\mathrm{Wh} / \mathrm{m}^{2}$, which is the final output shown by the augmented virtuality solar energy calculator as shown in Figures 4 and 5. 


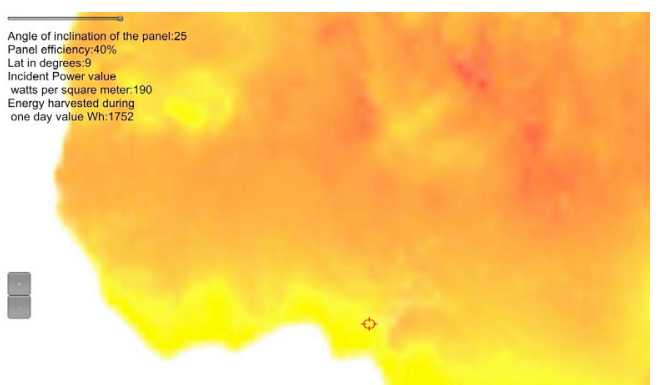

Figure 5. Example of augmented virtuality solar energy calculator in action

\section{E. Discussion}

The augmented virtuality solar energy power calculator on start up presents the learner with a solar irradiance world map as the environment for all input and output from the application. The included zoom controls are used to enhance the precision of the input location and subsequently the calculated power output. The application is interactive and requires the learner to indicate by touching the approximate location of interest, and then the application will provide the geographical latitude and the solar irradiance value corresponding to these coordinates. The operational panel efficiency (with a range between 5 and 40) is entered from a slider control located in the upper left section of the display and may be used to show the difference in output between different models of solar panels and devices from different manufacturers.

The real-time value of the physical inclination of the mobile device is read from the mobile device's hardware accelerometer. All four values are then used to calculate the mean power output during one day in $\mathrm{W} / \mathrm{m}^{2}$, this is subsequently multiplied by 24 to obtain the total energy captured during one day in $\mathrm{Wh} / \mathrm{m}^{2}$ and show on the display as "Energy harvested during one day" along with the values of all other variable used.

Additionally, as the results are presented immediately, the learner is able to quickly use the application in comparing the power outputs at different sites/locations, which is useful when identifying suitable sites for large scale solar energy power generation farms.

\section{CONCLUSION}

This paper has presented an augmented virtuality tool that runs on mobile devices equipped with appropriate sensor hardware. The solar panel energy output calculator provides hands-on introduction to some practical aspects of solar power energy generation and compliments existing education resources. The interactive nature of the application ensures an improved learning experience with the ability to quickly estimate and compare solar energy power output for various locations, inclinations and device models. The tool is cost effective especially if used on personal mobile devices of learners and may also be used as a completely off-line aid or companion tool for the informal training of engineers and professionals. Although the power output value from the tool is an approximation, it is capable of results that highlight subtle differences that may occur with different brands/models of solar panels. It should be noted that the resolution and therefore location accuracy obtainable from the world solar irradiance map is limited. In the field of solar energy power generation and photovoltaic, this tool enhances several learning outcomes in a practical and hands-on manner that ensures that learners (engineer and professional) are better equipped with the skills for meeting the challenges and demands imposed by the increasing importance of solar energy as a viable source of generating electrical power.

\section{ACKNOWLEDGMENT}

The augmented virtuality component and android application is based on the prior work and contributions from Engineer. M. Santachiara of Santa's Co, Reggio Emilia, Italy.

\section{REFERENCES}

[1] EPIA, "Global Market Outlook for Photovoltaics 2013-2017," European Photovoltaic Industry Association, Brussels, Technical Report 2013.

[2] Faisal Ahammed and Azeem Abdullahil, "An economic analysis of solar PV micro-utility in rural areas of Bangladesh," in 1st International Conference on Developments in Renewable Energy, Dhaka, 2009, pp. 1-4..

[3] P Bauer, A Rojko, and R. Ionel, "Distance Learning Module for Solar Electricity with Programming of MPPT," in 15th European Conference on Power Electronics and Applications, Lille, 2013, pp. 1-8.

[4] C Onime and J Uhomoibhi, "Engineering Education in a developing country: Experiences from Africa," in 15th International Conference on Interactive Collaborative Learning (ICL), Klagenfuth, 2012, pp. 1-3.

[5] D. A Umphress, d. Hendrix, and J. H Cross, "Software processes in the classroom: The capstone project experience," IEEE Software, vol. 19, no. 5, pp. 78-85, Sep.-Oct. 2002. http://dx.doi.org/10.1109/MS.2002.1032858

[6] Narayanan Komerath and Marilyn Smith, "Research Seminars as a Resource in Mentoring Undergraduates," in Innovations 2011: World Innovations in Engineering Education and Research, W Aung et al., Eds. Arlington: iNEER, 2011, pp. 183-193..

[7] A Rojko and K Kozlowski, "Lifelong education in robotics and mechatronics ," in 17th International Conference on Methods and Models in Automation and Control, Miedzyzdrojie , 2012, pp. 343-348.

[8] R. Koseler, S. Shapcott, K.,G Nelson, and J Husman, "Work in progress: Evaluation of an online education portal from the user's perspective: An empirical investigation of a photovoltaics (PV) engineering learning portal, pveducation.org," in Frontiers in Education Conference, Seattle, WA, 2012, pp. 1-6.

[9] E da S Brito et al., "Comparison of Solar Panel models for Grid Integration Studies," in 10th IEEE/IAS International Conference on Industry Applications (INDUSCON), 2012, pp. 1-8.

[10] 3TIER. (2014, January) Free Renewable Energy Reosurce Maps: Wind Speed, Solar Irradiance, Hydropower. [Online]. http://www.3tier.com/en/support/resource-maps/

\section{AUTHORS}

C. Onime is with the Abdus Salam International Centre for Theoretical Physics, Strada Costiera 11, Trieste, 34100, Italy (e-mail: clement.onime@ictp.it).

E. Pietrosemoli is with Abdus Salam International Centre for Theoretical Physics, Strada Costiera 11, Trieste, 34100, Italy (e-mail: pietrosemoli@ictp.it).

J. Uhomoibhi is with the University of Ulster, Antrim Road, Newtownabbey, Belfast, Northern Ireland (e-mail: j.uhomoibhi@ulster.ac.uk).

This work was jointly supported by the Telecommunications/ICT for Development (T/ICT4D) Laboratory and the Information, Communication and Telecommunications Section (ICTS), both of the Abdus Salam International Centre for Theoretical Physics (ICTP). Submitted 06 May 2014. Published as resubmitted by the authors 14 February 2015. 